\title{
Oscillation for Super Linear/Linear Second Order Neutral Difference Equations with Variable Several Delays
}

\author{
Chittaranjan Behera \\ Department of Mathematics, \\ Silicon Institute of Technology, Bhubaneswar, Odisha, India. \\ Corresponding author: crb_sit@yahoo.co.uk \\ Radhanath Rath \\ Department of Mathematics, \\ Veer Surendra Sai University of Technology, Burla, Odisha, India; \\ Flat A203, Center Point Apartment, Bhubaneswar, Odisha, India. \\ E-mail: radhanathmath@yahoo.co.in \\ Prayag Prasad Mishra \\ Department of Mathematics, \\ Silicon Institute of Technology, Bhubaneswar, Odisha, India. \\ E-mail: prayag@silicon.ac.in
}

(Received November 21, 2019; Accepted March 20, 2020)

\begin{abstract}
This article, is concerned with finding sufficient conditions for the oscillation and non oscillation of the solutions of a second order neutral difference equation with multiple delays under the forward difference operator, which generalize and extend some existing results. This could be possible by extending an important lemma from the literature.
\end{abstract}

Keywords- Oscillation, Non oscillation, Neutral difference equation, Asymptotic behavior.

\section{Introduction}

This article is concerned with finding sufficient conditions so that a solution of the neutral delay difference equation(NDDE in short)

$\Delta^{2}\left(y_{n}-\sum_{j=1}^{k} p_{n}^{\{j\}} y_{n-m_{j}}\right)+v_{n} G\left(y_{\sigma(n)}\right)=f_{n}$

which does not oscillate, tends to zero as $n \rightarrow \infty$. Here $\Delta$ is the forward difference operator, given by $\Delta x_{n}=x_{n+1}-x_{n}, p_{n}^{\{j\}}, v_{n}$ and $f_{n}$ are members of infinite sequences of real numbers with $v_{n}>0, G \in C(R, R)$. Further, we assume $\{\sigma(n)\}$ is an unbounded sequence such that $\sigma(n) \leq n$ for every $n$. Different ranges of the $\left\{p_{n}^{\{j\}}\right\}$ for $j=1,2, \ldots, k$ are considered. The $m_{j}$ for $j=$ $1,2, \ldots, k$ are positive integers.

The following hypothesis are needed in the sequel,

(E1) $x G(x)>0$ for $x \neq 0$.

(E2) $v_{n}>0, \sum_{n=n_{0}}^{\infty} v_{n}=\infty$.

(E3) There exists $\left\{F_{n}\right\}$, a bounded sequence such that $\Delta^{2} F_{n}=f_{n}$. 
(E4) The sequence $F_{n}$ in (E3) satisfies $\lim _{n \rightarrow \infty} F_{n}=0$.

(E5) $\sum_{n_{0}}^{\infty} v_{n}^{*}=\infty$, where $v_{n}^{*}=\min \left\{v_{n}, v_{n-m_{1}}, v_{n-m_{2}}\right\}$.

(E6) For $v>0, w>0, u>0$, there exists a scalar $\beta>0$, such that $G(v) G(w) \geq G(v w)$ and

$$
G(v)+G(w)+G(u) \geq \beta G(v+w+u)
$$

(E7) $\sum_{n=n_{0}}^{\infty} n v_{n}=\infty$.

(E8) $\sum_{j=1}^{\infty}\left(n_{j}\right) v_{n_{j}}=\infty \quad$ where $v_{n j}$ is any subsequence of $v_{n}$.

(E9) For $u>0$ there exists $\delta>0$ such that $G(u) \geq \delta u$. For $u<0$ there exists $\delta>0$ such that $G(u) \leq \delta u$.

(E10) $G(-u)=-G(u)$.

(E11) $\liminf _{n \rightarrow \infty} \sigma(n) / n>0$.

Remark 1.1 By (E9), if $\liminf _{n \rightarrow \infty} u_{n}>0$ then $\exists \delta>0$ such that $\liminf _{n \rightarrow \infty} G\left(u_{n}\right) / u_{n}>\delta$. We assume that $p_{n}^{\{j\}}, j=1,2, \ldots, k$ are bounded and satisfies one of the following conditions. There exists positive constants $b_{j}, j=1,2, \ldots, k$ and $b$ such that

(R1) $b_{j} \geq p_{n}^{\{j\}} \geq 0, \forall \quad j=1,2, \ldots k \quad$ and $\sum_{j=1}^{k} \liminf _{\mathrm{n} \rightarrow \infty} p_{n}^{\{j\}}<\sum_{j=1}^{k} b_{j}=b<1$.

(R2) $-b_{j} \leq p_{n}^{\{j\}} \leq 0, \forall \quad j=1,2, \ldots k$ and $\sum_{j=1}^{k} \liminf _{\mathrm{n} \rightarrow \infty} p_{n}^{\{j\}} \geq \sum_{j=1}^{k}-b_{j}=-b>$

$$
p_{n}^{\{j\}} \leq 0, \forall j=1,2, \ldots k \text { and } \exists i \in\{1,2,3, \ldots, k\} \text { such that }
$$

(R3) $\limsup p_{n}^{\{i\}}-\sum_{j \neq i} \liminf p_{n}^{\{j\}}<-1$.

$$
p_{n}^{\{j\}} \geq 0 \quad \forall \quad j=1,2, \ldots k \text { and } \exists i \in\{1,2,3, \ldots, k\} \text { such that }
$$

(R4) $\liminf p_{n}^{\{i\}}-\sum_{j \neq i} \limsup p_{n}^{\{j\}}>1$.

For easy understanding and convenience of writing the proofs of the results, the higher order NDDE

$\Delta^{2}\left(y_{n}-p_{n}^{\{1\}} y_{n-m_{1}}-p_{n}^{\{2\}} y_{n-m_{2}}\right)+v_{n} G\left(y_{\sigma(n)}\right)=f_{n}$

with two delay terms under the $\Delta^{2}$ sign, is considered, instead of (1) and this is with out any loss of generality.

One of the following conditions are to be assumed on the bounded sequences $\left\{p_{n}^{\{j\}}\right\}$ for $j=1,2$ while considering the neutal equation (2).

There exists positive constants $b, b_{1}$, and $b_{2}$ such that 
(R5)

$$
b \geq p_{n}^{\{1\}}>0, b \geq p_{n}^{\{2\}} \geq 0
$$

(R6) $-b \leq p_{n}^{\{1\}}<0,-b \leq p_{n}^{\{2\}} \leq 0$.

$$
1>b_{1} \geq p_{n}^{\{1\}} \geq 0,1>b_{2} \geq p_{n}^{\{2\}} \geq 0 \text {, and } b_{1}+b_{2}=b<1 .
$$

(R8) $-1<-b_{1} \leq p_{n}^{\{1\}} \leq 0,-1<-b_{2} \leq p_{n}^{\{2\}} \leq 0$ and $b_{1}+b_{2}=b<1$.

(R9) $b \geq p_{n}^{\{1\}}>1, b \geq p_{n}^{\{2\}} \geq 0$.

(R10) $-b \leq p_{n}^{\{1\}}<-1,-b \geq p_{n}^{\{2\}} \leq 0$.

Note that (R1) and (R2) are equivalent to (R7) and (R8) respectively. Further note that (R6) is less restrictive than (R3) and (R5) is less restrictive than (R4). If $p_{n}=p_{n}^{\{1\}}$, then the ranges of $p_{n}$, which are obtained, by the substitution $\mathrm{k}=1$, in $(\mathrm{R} 1)-(\mathrm{R} 4)$, or $p_{n}^{\{2\}}=0$, in (R7) - (R10), are considered (Parhi and Tripathy, 2003; Rath et al., 2010).

Let $N_{1}$ be a fixed non negative integer and $r=\max \left\{m_{j}: j=1,2, \ldots, k\right\}$. Let $N_{0}=\min \left\{N_{1}-\right.$ $\left.r, \sigma\left(N_{1}\right)\right\}$. A solution of (1), is defined as "a real sequence $\left\{y_{n}\right\}$, which is defined $\forall+$ ve integer $n \geq N_{0}$, and satisfies (1) for $n \geq N_{1}$. Further, if the initial values

$y_{n}=a_{n}$ for $N_{0} \leq n \leq N_{1}+1$,

are provided then the equation (1) has a unique solution satisfying the initial values (3). A non trivial solution $\left\{y_{n}\right\}$ of (1) is called oscillatory, if for any positive integer $n_{0} \geq N_{1}$, there exists $n \geq n_{0}$ such that $y_{n} y_{n+1} \leq 0$, otherwise $\left\{y_{n}\right\}$ is said to be non-oscillatory."

As is well known that, it is not always easy to solve a functional difference equation and find it's solution in closed form, therefore, qualitative theory of difference equations is developed rapidly, since here we assume that the solutions of the difference equation exist and concentrate to investigate its oscillatory behaviour . Recently, numerous articles on oscillation of solutions of neutral difference equations are published for example (Agarwal et al., 1996; Agarwal and Grace, 1999; Parhi and Tripathy, 2003; Zhou and Huang, 2003; Yildz and Ocalan, 2007; Karpuz et al., 2009a; Karpuz et al., 2009b; Yildiz et al., 2009; Yildiz, 2015) and the references cited therein. Thandapani et al. (1999) found non-oscillation and oscillation criteria for the equation

$\Delta^{m}\left(y_{n}-p_{n} y_{n-l}\right)+v_{n} G\left(y_{n-r}\right)=f_{n}$.

Here, we study the oscillatory behaviour of (1) and (2), which seems, not being considered by any author till date. This paper generalizes the study of the equation (4) for $m=2$. We observe that while studying the NDDEs, the authors (Parhi and Tripathy, 2003; Rath and Padhy, 2005; Rath et al., 2010) have significantly used the Lemma 2.1 of Parhi and Tripathy (2003), which is the discrete analogue to the Lemma 1.5.2 of Gyori and Ladas (1991), for their results. It is further observed that, even, many results for the study of neutral differential equations (i.e; the continuous case) are dependent on a similar result, i.e; Lemma 1.5.2 of Gyori and Ladas (1991). However, the Lemma 
International Journal of Mathematical, Engineering and Management Sciences

Vol. 5, No. 4, 663-681, 2020

https://doi.org/10.33889/IJMEMS.2020.5.4.054

2.1 of Parhi and Tripathy (2003) cannot be applied to the study of (1) or that of (2). In this context, one may go through the "open problem 1.8, at page 31 of Gyori and Ladas (1991) which suggests to extend the lemma suitably, for its own sake and its application to the study of the neutral equations with several delays." In this article we extend the lemma for the said purpose in order to study the oscillatory behavior of (1) or (2), there by, improving, extending and generalizing some results of Parhi and Tripathy (2003); Rath and Behera (2018).

\section{Some Lemmas}

In this section first, we quote some results from different research articles, that would be helpful in the sequel.

Lemma 2.1 (Parhi and Tripathy, 2003) [Lemma 2.1] "Let $\left\{f_{n}\right\},\left\{q_{n}\right\}$ and $\left\{p_{n}\right\}$ be real sequences defined for $n \geq N_{0}>0$ such that

$f_{n}=q_{n}-p_{n} q_{n-m}, n \geq N_{0}+m$

where $m \geq 0$ is an integer. Let $b, b_{1}$ and $b_{2}$ be reals such that $p_{n}$ satisfies one of the three conditions below

(i) $-1<-b \leq p_{n} \leq 0$, (ii) $-b_{2} \leq p_{n} \leq-b_{1}<-1$, (iii) $0 \leq p_{n} \leq b_{2}$.

If $q_{n}>0$ for $n>N_{0}, \liminf _{\mathrm{n} \rightarrow \infty} q_{n}=0$ and $\lim _{n \rightarrow \infty} f_{n}=\delta$ exists then $\delta=0$."

Lemma 2.2 (Royden, 1988) " Let $\left\{u_{n}\right\}$ and $\left\{v_{n}\right\}$ be two real sequences defined for $n \geq n_{0}>0$. Then

$\lim \inf _{n \rightarrow \infty} u_{n}+\lim \inf _{n \rightarrow \infty} v_{n} \leq \lim \inf _{n \rightarrow \infty}\left(u_{n}+v_{n}\right) \leq \limsup u_{n \rightarrow \infty}+\lim \inf _{n \rightarrow \infty} v_{n}$

(or $\left.\quad \liminf _{n \rightarrow \infty} u_{n}+\limsup _{n \rightarrow \infty} v_{n}\right) \leq \limsup _{n \rightarrow \infty}\left(u_{n}+v_{n}\right) \leq \limsup _{n \rightarrow \infty} u_{n}+\limsup _{n \rightarrow \infty} v_{n}$

provided that no sum is of the form $\infty-\infty$."

Lemma 2.3 (Royden, 1988) " Let $\left\{u_{n}\right\}$ and $\left\{v_{n}\right\}$ be two non negative real sequences defined for $n \geq n_{0}>0$. Then

$\lim \inf _{n \rightarrow \infty} u_{n} \times \lim \inf _{n \rightarrow \infty} v_{n} \leq \lim \inf _{n \rightarrow \infty}\left(u_{n} \times v_{n}\right) \leq \limsup u_{n \rightarrow \infty} u_{n} \times \lim \inf _{n \rightarrow \infty} v_{n}$

(or $\left.\quad \lim \inf _{n \rightarrow \infty} u_{n} \times \limsup v_{n \rightarrow \infty} v_{n}\right) \leq \limsup \left(u_{n} \times v_{n}\right) \leq \limsup _{n \rightarrow \infty} u_{n} \times \limsup v_{n \rightarrow \infty} v_{n}$

provided that no product is of the form $0 \times \infty$."

Lemma 2.4 (Agarwal, 2000; Parhi and Tripathy, 2003) "Let $z_{n}$ be a real valued function defined for $n \in N\left(n_{0}\right)=\left\{n_{0}, n_{0}+1, \ldots\right\}, n_{0}>0$ and $z_{n}>0$ with $\Delta^{m} z_{n}$ either + ve or $-v e$ on $N\left(n_{0}\right)$ and not equal to zero.Then $\exists$ an integer $p, 0 \leq p \leq m-1$, with $(m+p)$ even for $\Delta^{m} z_{n} \geq$ 0 , and $m+p$ odd for $\Delta^{m} z_{n} \leq 0$ such that 
International Journal of Mathematical, Engineering and Management Sciences

Vol. 5, No. 4, 663-681, 2020

https://doi.org/10.33889/IJMEMS.2020.5.4.054

$\Delta^{i} z_{n}>0 \quad$ for $\quad n \geq n_{0}, 0 \leq i \leq p$,

$(-1)^{p+i} \Delta^{i} z_{n}>0, \quad$ for $\quad n \geq n_{0}, p+1 \leq i \leq m-1$."

Remark 2.5 From the above lemma, for $m=2$, if $\Delta^{2} z_{n} \leq 0$ and $z_{n} \geq 0$ then $p=1$ and $\Delta z_{n}>$ 0 .

Definition 2.6 "Define the factorial function (Kelley and Peterson, 1991) by $n^{(k)}:=$ $n(n-1) \ldots(n-k+1)$,

where $k \leq n$ and $n \in Z$ and $k \in N$. Note that $n^{(k)}=0$, if $k>n$."

Lemma 2.7 (Rath, et al., 2010) "Let $p \in N$ and $x(n)$ be a +ve real sequence in $\left[n_{1}, \infty\right)$ for some large $n_{1}$. If $\exists$ an integer $p_{0} \in\{0,1, \ldots, p-1\}$, such that $\Delta^{i} w(\infty)=0$ and $\Delta^{p_{0}} w(\infty)$ exits (finite) for all $i \in\left\{p_{0}+1, \ldots, p-1\right\}$. Then

$\Delta^{p} w(n)=-x(n)$

implies

$\Delta^{p_{0}} w(n)=\Delta^{p_{0}} w(\infty)+\frac{(-1)^{p-p_{0}-1}}{\left(p-p_{0}-1\right) !} \sum_{i=n}^{\infty}\left(i+p-p_{0}-1-n\right)^{\left(p-p_{0}-1\right)} x(i)$,

for all sufficiently large $n$."

Remark 2.8 Consider $\left\{w_{n}\right\}$ as a real sequence and $L$ as a +ve scalar such that $w_{n}>L$ for $n \geq$ $n_{1}$. If $z_{n} \geq w_{n}-\epsilon$ for $n \geq n_{2} \geq n_{1}$, where $\epsilon$ is is any arbitrary pre assigned positive number, then $\exists \mathrm{a}+$ ve scalar $C<L$ and a +ve integer $n_{3} \geq n_{2}$ such that $n \geq n_{3}$ implies $z_{n} \geq C$.

Lemma 2.9 (Malik and Arora, 2008) "If $\sum \mathrm{u}_{\mathrm{n}}$ and $\sum \mathrm{v}_{\mathrm{n}}$ are two positive term series such that $\lim _{n \rightarrow \infty}\left(\frac{u_{n}}{v_{n}}\right)=1$, where $l$ is a finite number and not equal to zero, then the two series diverge or converge together. If $\mathrm{l}=\infty$ then divergence of $\sum \mathrm{v}_{\mathrm{n}} \Rightarrow$ divergence of $\sum \mathrm{u}_{\mathrm{n}}$. If $\mathrm{l}=0$ then, convergence of $\sum \mathrm{v}_{\mathrm{n}} \Rightarrow$ convergence of $\sum \mathrm{u}_{\mathrm{n}}$."

Remark 2.10 By Lemma 2.9, it follows that (E7) holds if and only if $\sum_{n=n_{0}}^{\infty}\left(n-n_{0}+1\right) v_{n}=\infty$. It is because $(n-r+1)^{r}<n^{(r)}<n^{r}$ for $r \leq n$.

Remark 2.11 The condition $\left|\sum_{n=n_{0}}^{\infty} n f_{n}\right|<\infty$ implies that (E3) and (E4) holds.In fact, if we define $F_{n}=\sum_{j=n}^{\infty}(j-n+1) f_{j} \quad$ by Lemma 2.9 then, $\Delta^{2} F_{n}=f_{n}$ and $\lim _{n \rightarrow \infty} F_{n}=0$.

The following result extends and generalizes the Lemma 2.1.

Lemma 2.12 Assume $y_{n}>0$ for $n \geq n_{0}$ with limin $f_{n \rightarrow \infty} y_{n}=0$. Suppose that $z_{n}=y_{n}-\sum_{j=1}^{k} p_{n}^{\{j\}} y_{n-m_{j}}$. 
Further, assume that $\lim _{n \rightarrow \infty} z_{n}=\delta$ exists finitely. Then

(a) If $p_{n}^{\{j\}} \geq 0$ then $\delta \leq 0$ and if $p_{n}^{\{j\}} \leq 0$ then $\delta \geq 0$.

(b) Further, suppose that $y_{n}$ is bounded and $p_{n}^{\{j\}}, j=1,2, \ldots, k$, satisfy one of the four conditions (R1), (R2), (R3) or (R4). Then $\delta=0$ and $\lim _{n \rightarrow \infty} y_{n}=0$.

Proof. (a) Since $\lim _{n \rightarrow \infty} z_{n}=\delta$ exists finitely then $\liminf _{n \rightarrow \infty} Z_{n}=\limsup _{n \rightarrow \infty} z_{n}=\delta$. If $p_{n}^{\{j\}} \geq$ 0 then $z_{n} \leq y_{n}$ and $\liminf _{n \rightarrow \infty} z_{n} \leq \liminf _{n \rightarrow \infty} y_{n}$. This implies $\delta \leq 0$. Again if $p_{n}^{\{j\}} \leq 0$ then $z_{n} \geq y_{n}$ and this implies $\delta \geq 0$. Hence the result follows.

(b) Since $y_{n}$ is bounded then $\liminf _{n \rightarrow \infty} y_{n}$ and $\limsup _{n \rightarrow \infty} y_{n}$ exists finitely.

Let us consider case (i) i.e; $p_{n}^{\{j\}}$ satisfy(R1). This implies $p_{n}^{\{j\}} \geq 0$. Hence we obtain $\delta \leq 0$. Then using Lemma 2.2 and 2.3 we have

$$
\begin{aligned}
& 0 \geq \delta=\limsup _{n \rightarrow \infty} z_{n}=\limsup _{n \rightarrow \infty}\left(y_{n}-\sum_{j=1}^{k} p_{n}^{\{j\}} y_{n-m_{j}}\right) \\
& \geq \limsup _{n \rightarrow \infty} y_{n}+\liminf _{n \rightarrow \infty}\left(-\sum_{j=1}^{k} p_{n}^{\{j\}} y_{n-m_{j}}\right) \\
& \geq \limsup _{n \rightarrow \infty} y_{n}-\limsup _{n \rightarrow \infty}\left(\sum_{j=1}^{k} p_{n}^{\{j\}} y_{n-m_{j}}\right) \\
& \geq \limsup _{n \rightarrow \infty} y_{n}-\sum_{j=1}^{k} \limsup _{n \rightarrow \infty}\left(p_{n}^{\{j\}} y_{n-m_{j}}\right) \\
& \geq \limsup _{n \rightarrow \infty} y_{n}-\sum_{j=1}^{k} \limsup _{n \rightarrow \infty} p_{n}^{\{j\}} \limsup _{n \rightarrow \infty} y_{n-m_{j}} \\
& \geq \limsup _{n \rightarrow \infty} y_{n}\left(1-\sum_{j=1}^{k} \limsup _{n \rightarrow \infty} p_{n}^{\{j\}}\right) \\
& \geq \limsup _{n \rightarrow \infty} y_{n}(1-b) \geq 0 .
\end{aligned}
$$

Hence $\delta=0$ and $\limsup _{n \rightarrow \infty} y_{n} \leq 0$, by (R1), which implies $\limsup _{n \rightarrow \infty} y_{n}=0$. Hence $\lim _{n \rightarrow \infty} z_{n}=0$ and $\lim _{n \rightarrow \infty} y_{n}=0$.

Next consider case (ii) i.e; $p_{n}^{\{j\}}$ satisfy(R2). Clearly, $z_{n} \geq y_{n}$ due to (R2) and this implies $\delta \geq 0$. Further, using Lemma 2.2 and 2.3 we have

$$
\begin{aligned}
\delta= & \liminf _{n \rightarrow \infty} z_{n}=\liminf _{n \rightarrow \infty}\left(y_{n}-\sum_{j=1}^{k} p_{n}^{\{j\}} y_{n-m_{j}}\right) \\
& \leq \liminf _{n \rightarrow \infty} y_{n}+\limsup _{n \rightarrow \infty}\left(\sum_{j=1}^{k}-p_{n}^{\{j\}} y_{n-m_{j}}\right) \\
& \leq \sum_{j=1}^{k} \limsup _{n \rightarrow \infty}\left(-p_{n}^{\{j\}}\right)\left(y_{n-m_{j}}\right)
\end{aligned}
$$




$$
\begin{aligned}
& \leq \sum_{j=1}^{k} \limsup _{n \rightarrow \infty}\left(-p_{n}^{\{j\}}\right) \limsup _{n \rightarrow \infty}\left(y_{n-m_{j}}\right) \\
& \leq \sum_{j=1}^{k}-\liminf _{n \rightarrow \infty}\left(p_{n}^{\{j\}}\right) \limsup _{n \rightarrow \infty}\left(y_{n-m_{j}}\right) \\
& \leq b \limsup _{n \rightarrow \infty} y_{n} \leq b \alpha .
\end{aligned}
$$

Hence we get

$\alpha \geq \frac{\delta}{b}>\delta$.

Again

$$
\begin{aligned}
\delta= & \limsup _{n \rightarrow \infty} z_{n}=\limsup _{n \rightarrow \infty}\left(y_{n}-\sum_{j=1}^{k} p_{n}^{\{j\}} y_{n-m_{j}}\right) \\
& \geq \limsup _{n \rightarrow \infty} y_{n}+\liminf _{n \rightarrow \infty}\left(-\sum_{j=1}^{k} p_{n}^{\{j\}} y_{n-m_{j}}\right) \\
& \geq \limsup _{n \rightarrow \infty} y_{n}+\liminf _{n \rightarrow \infty}\left(\sum_{j=1}^{k}\left(-p_{n}^{\{j\}}\right) y_{n-m_{j}}\right) \\
& \geq \underset{n \rightarrow \infty}{\limsup } y_{n}+\sum_{j=1}^{k} \liminf _{n \rightarrow \infty}\left(\left(-p_{n}^{\{j\}}\right) y_{n-m_{j}}\right) \\
& \geq \limsup _{n \rightarrow \infty} y_{n}+\sum_{j=1}^{k} \liminf _{n \rightarrow \infty}\left(-p_{n}^{\{j\}}\right) \liminf _{n \rightarrow \infty} y_{n-m_{j}} \\
& \geq \limsup _{n \rightarrow \infty} y_{n}=\alpha .
\end{aligned}
$$

Combining the above inequation with (9), it follows that $\alpha>\delta \geq \alpha$, a contradiction which implies $\delta=0=\alpha$.

Let us consider case iii: i.e; $p_{n}^{\{j\}}$ satisfy(R3). Clearly, $z_{n} \geq y_{n}$ due to (R3) and this implies $\delta \geq 0$. Further, using Lemma 2.2 and 2.3 we have

$$
\begin{aligned}
\delta= & \liminf _{n \rightarrow \infty} z_{n}=\liminf _{n \rightarrow \infty}\left(y_{n}-\sum_{j=1}^{k} p_{n}^{\{j\}} y_{n-m_{j}}\right) \\
& \leq \limsup _{n \rightarrow \infty}\left(y_{n}+\sum_{j \neq i}-p_{n}^{\{j\}} y_{n-m_{j}}\right)+\liminf _{n \rightarrow \infty}\left(-p_{n}^{\{i\}} y_{n-m_{i}}\right) \\
& \leq \limsup _{n \rightarrow \infty}+\limsup _{n \rightarrow \infty} \sum_{j \neq i}-p_{n}^{\{j\}} y_{n-m_{j}}+\underset{n \rightarrow \infty}{\limsup }\left(-p_{n}^{\{i\}}\right) \liminf _{n \rightarrow \infty}\left(y_{n-m_{i}}\right) \\
& \leq \limsup _{n \rightarrow \infty}+\sum_{j \neq i} \limsup _{n \rightarrow \infty}\left(-p_{n}^{\{j\}} y_{n-m_{j}}\right) \\
& \leq \limsup _{n \rightarrow \infty}+\sum_{j \neq i} \limsup _{n \rightarrow \infty}\left(-p_{n}^{\{j\}}\right) \limsup _{n \rightarrow \infty}\left(y_{n-m_{j}}\right) \\
& \leq \limsup _{n \rightarrow \infty}\left(y_{n}\right)\left[1-\sum_{j \neq i} \liminf _{n \rightarrow \infty} p_{n}^{\{j\}}\right] .
\end{aligned}
$$


International Journal of Mathematical, Engineering and Management Sciences

Vol. 5, No. 4, 663-681, 2020

https://doi.org/10.33889/IJMEMS.2020.5.4.054

Again, we have

$$
\begin{aligned}
\delta= & \limsup _{n \rightarrow \infty} z_{n}=\limsup _{n \rightarrow \infty}\left(y_{n}-\sum_{j=1}^{k} p_{n}^{\{j\}} y_{n-m_{j}}\right) \\
& \geq \liminf _{n \rightarrow \infty} y_{n}+\limsup _{n \rightarrow \infty}\left(-\sum_{j=1}^{k} p_{n}^{\{j\}} y_{n-m_{j}}\right) \\
& \geq 0+\limsup _{n \rightarrow \infty}\left(-\sum_{j=1}^{k} p_{n}^{\{j\}} y_{n-m_{j}}\right) \\
& \geq \limsup _{n \rightarrow \infty}\left(-p_{n}^{\{i\}} y_{n-m i}\right)+\liminf _{n \rightarrow \infty} \sum_{j \neq i}\left(-p_{n}^{\{j\}} y_{n-m_{j}}\right) \\
& \geq \limsup _{n \rightarrow \infty} y_{n-m_{i}} \liminf _{n \rightarrow \infty}\left(-p_{n}^{\{i\}}\right)+\sum_{j \neq i} \liminf _{n \rightarrow \infty}\left(\left(-p_{n}^{\{j\}}\right) y_{n-m_{j}}\right) \\
& \geq \limsup _{n \rightarrow \infty} y_{n-m_{i}} \liminf _{n \rightarrow \infty}\left(-p_{n}^{\{i\}}\right)+\sum_{j \neq i}\left(\liminf _{n \rightarrow \infty}\left(-p_{n}^{\{j\}}\right) \liminf _{n \rightarrow \infty} y_{n-m_{j}}\right) \\
& \geq \limsup _{n \rightarrow \infty} y_{n}\left(-\limsup _{n \rightarrow \infty} p_{n}^{\{i\}}\right) .
\end{aligned}
$$

From (10) and (11), it follows that

$$
\underset{n \rightarrow \infty}{\limsup } y_{n}\left(\left(\sum_{j \neq i} \liminf _{n \rightarrow \infty} p_{n}^{\{j\}}\right)-1-\limsup _{n \rightarrow \infty} p_{n}^{\{i\}}\right) \leq 0 \text {. }
$$

Using (R3), we obtain $\limsup _{n \rightarrow \infty} y_{n}=\alpha=0$. Then from (10) and (11) we have $\delta \leq 0$ and $\delta \geq$ 0 respectively. This implies $\lim _{n \rightarrow \infty} z_{n}=0$ and $\lim _{n \rightarrow \infty} y_{n}=0$.

Let us consider case (iv) i.e; $p_{n}^{\{j\}}$ satisfy (R4). Then $p_{n}^{\{j\}} \geq 0$ and $\delta \leq 0$. By Lemma 2.2 and 2.3, we have

$$
\begin{aligned}
\delta= & \liminf _{n \rightarrow \infty} z_{n}=\liminf _{n \rightarrow \infty}\left(y_{n}-\sum_{j=1}^{k} p_{n}^{\{j\}} y_{n-m_{j}}\right) \\
& \leq \limsup _{n \rightarrow \infty} y_{n}+\liminf _{n \rightarrow \infty} \sum_{j=1}^{k}-p_{n}^{\{j\}} y_{n-m_{j}} \\
& \leq \alpha+\liminf _{n \rightarrow \infty}\left(-p_{n}^{\{i\}} y_{n-m_{i}}\right)+\limsup _{n \rightarrow \infty} \sum_{j \neq i}-p_{n}^{\{j\}} y_{n-m_{j}} \\
& \leq \alpha-\limsup _{n \rightarrow \infty}\left(p_{n}^{\{i\}} y_{n-m_{i}}\right)+\sum_{j \neq i} \limsup _{n \rightarrow \infty}\left(-p_{n}^{\{j\}} y_{n-m_{j}}\right) \\
& \leq \alpha-\liminf _{n \rightarrow \infty} p_{n}^{\{i\}} \limsup _{n \rightarrow \infty} y_{n-m_{i}}-\left(\sum_{j \neq i} \liminf _{n \rightarrow \infty} p_{n}^{\{j\}} y_{n-m_{j}}\right) \\
& \leq \alpha-\alpha \liminf _{n \rightarrow \infty} p_{n}^{\{i\}}-\left(\sum_{j \neq i} \liminf _{n \rightarrow \infty} p_{n}^{\{j\}} \liminf _{n \rightarrow \infty} y_{n-m_{j}}\right) \\
& \leq \alpha\left(1-\liminf _{n \rightarrow \infty} p_{n}^{\{i\}}\right)
\end{aligned}
$$


Again we have

$$
\begin{aligned}
& \delta=\limsup _{n \rightarrow \infty} z_{n}=\limsup _{n \rightarrow \infty}\left(y_{n}-\sum_{j=1}^{k} p_{n}^{\{j\}} y_{n-m_{j}}\right) \\
& \geq \liminf _{n \rightarrow \infty} y_{n}+\limsup _{n \rightarrow \infty}\left(-\sum_{j=1}^{k} p_{n}^{\{j\}} y_{n-m_{j}}\right) \\
& \geq \limsup _{n \rightarrow \infty}\left(-p_{n}^{\{i\}} y_{n-m_{i}}\right)+\liminf _{n \rightarrow \infty} \sum_{j \neq i}\left(-p_{n}^{\{j\}} y_{n-m_{j}}\right) \\
& \geq-\liminf _{n \rightarrow \infty}\left(p_{n}^{\{i\}} y_{n-m_{i}}\right)-\sum_{j \neq i} \limsup _{n \rightarrow \infty}\left(p_{n}^{\{j\}} y_{n-m_{j}}\right) \\
& \geq-\liminf _{n \rightarrow \infty} y_{n-m_{i}}\left(\limsup _{n \rightarrow \infty} p_{n}^{\{i\}}\right)-\sum_{j \neq i}\left(\limsup _{n \rightarrow \infty} p_{n}^{\{j\}} \limsup _{n \rightarrow \infty} y_{n-m_{j}}\right) \\
& \geq-\limsup _{n \rightarrow \infty} y_{n}\left(\sum_{j \neq i} \limsup _{n \rightarrow \infty} p_{n}^{\{j\}}\right) \\
& \geq-\alpha\left(\sum_{j \neq i} \limsup _{n \rightarrow \infty} p_{n}^{\{j\}}\right) \text {. }
\end{aligned}
$$

From (12) and (13), it follows that

$-\alpha\left(\sum_{j \neq i} \limsup _{n \rightarrow \infty} p_{n}^{\{j\}}\right) \leq \delta \leq \alpha\left(1-\liminf _{n \rightarrow \infty} p_{n}^{\{i\}}\right)$.

By (R4), we obtain $\limsup _{n \rightarrow \infty} y_{n} \leq 0$. This implies $\limsup _{n \rightarrow \infty} y_{n}=0$. By (13), it follows that $\delta \geq 0$. Using part (a) of this lemma, we obtain $\delta=0$.Thus, the lemma is proved.

Lemma 2.13 Assume $y_{n}<0$ for $n \geq n_{0}$ with limsup $p_{n \rightarrow \infty} y_{n}=0$. Suppose that $z_{n}$ is defined as in (8).

Further, assume that $\lim _{n \rightarrow \infty} z_{n}=\delta$ exists finitely. Then

(a) If $p_{n}^{\{j\}} \geq 0$ then $\delta \geq 0$ and $p_{n}^{\{j\}} \leq 0$ then $\delta \leq 0$.

(b) Further, suppose that $y_{n}$ is bounded and $p_{n}^{\{j\}}, j=1,2, \ldots, k$, satisfy one of the four conditions (R1), (R2), (R3) or (R4). Then $\delta=0$ and $\lim _{n \rightarrow \infty} y_{n}=0$.

Proof: : The proof is similar to that of Lemma 2.12 and is therefore omitted.

Remark 2.14 The above Lemma 2.12 is an extension of Lemma 2.1. One may observe that $u_{n}$ and $v_{n}$ are not assumed to be bounded in Lemmas 2.1 or 2.2 or 2.3. However, it is assumed in Lemma 2.12 that $y_{n}$ and $y_{n-m_{j}}$ are bounded. This is only to avoid the conditions that "provided that no sum is of the form $\infty-\infty$ " in Lemma 2.2 and that "provided that no product is of the form $0 \times \infty$ " in Lemma 2.3. However, if each $p_{n}^{\{j\}}$, satisfies (R2) or (R3) then the terms in $z_{n}$ are positive when $y_{n}>0$. Hence in the limiting case the sum cannot be of the form $\infty-\infty$. Further, if $\liminf _{n \rightarrow \infty}\left|p_{n}^{j}\right|>0$, for each $\mathrm{j}$, in the case when (R2) holds, then the product term in Lemma 2.3 cannot be of the form $0 \times \infty$. Therefore, we can relax the condition of boundedness on $y_{n}$. In Lemma 2.12 and state it as another lemma. 
Lemma 2.15 Assume $y_{n}>0$ for $n \geq n_{0}$ with $\operatorname{limin} f_{n \rightarrow \infty} y_{n}=0$. Suppose that $z_{n}$ is defined as in (8) and that $\lim _{n \rightarrow \infty} z_{n}=\delta$ exists finitely. Let $p_{n}^{\{j\}}$ satisfy any one of the two conditions (R2) or (R3). Further, suppose that each $p_{n}^{\{j\}}$ satisfy $\liminf _{\mathrm{n} \rightarrow \infty}\left|p_{n}^{j}\right|>0$, if(R2) holds. Then $\delta=$ 0 and $\lim _{n \rightarrow \infty} y_{n}=0$.

Remark 2.16 Suppose $z_{n}$ is as defined in (8) with $k=2$. Then Lemmas 2.12, 2.13, and 2.15 hold if each $p_{n}^{\{j\}}$ satify one of the four conditions (R1), (R2), (R3), or (R4) with $k=2$.

Before the last lemma in this section is stated, it is assumed that $y=y_{n}$ is non-oscillatory solution of (2) for $n \geq N_{1}$. Define for $n \geq n_{0}$,

$z_{n}=y_{n}-p_{n}^{\{1\}} y_{n-m_{1}}-p_{n}^{\{2\}} y_{n-m_{2}}$

and

$w_{n}=z_{n}-F_{n}$

Lemma 2.17 Suppose that each $p_{n}^{\{j\}}$ satisfies the condition, (R4) with $k=2$, or (R9). Let (E1), (E3), (E4),(E7), (E9) and (E11) hold. Suppose that $y_{n}$ is a solution of (2) in some interval $\left[n_{1} \infty\right)$. Further assume that $z_{n}$ and $w_{n}$ as defined in (14) and (15) respectively. If $y_{n}>0$ then either $\lim _{n \rightarrow \infty} w_{n}=-\infty$ or $\lim _{n \rightarrow \infty} w_{n}=\lambda$ (finite) and $\lim _{n \rightarrow \infty} \Delta w_{n}=0$ with $\Delta w_{n}>0$. If $y_{n}<0$ then either $\lim _{n \rightarrow \infty} w_{n}=\infty$ or $\lim _{n \rightarrow \infty} w_{n}=\lambda$ (finite) and $\lim _{n \rightarrow \infty} \Delta w_{n}=0$ with $\Delta w_{n}<0$.

Proof. Let $y_{n}$ be an eventually positive solution of (2) for $n \geq n_{0} \geq N_{1}$. Then for $n \geq n_{0}$, using (14) and (15) in (2), we obtain

$\Delta^{2} w_{n}=-v_{n} G\left(y_{\sigma(n)}\right) \leq 0$.

Hence $w_{n}, \Delta w_{n}$ are monotonic for $n \geq n_{1}$ and of one sign. By (E3) and (E4) we have

$\lim _{n \rightarrow \infty} w_{n}=\lim _{n \rightarrow \infty} z_{n}=\lambda$, where $\lambda \in[-\infty, \infty]$.

If possible, let $\lambda$ be equal to $\infty$. Then $w_{n}>0$ and $\Delta w_{n}>0$ for $n \geq n_{1}$. Hence $\lim _{n \rightarrow \infty} \Delta w_{n}=l$, exists. Application of Lemma 2.7 to (16), for $n \geq n_{2}$ yields

$\Delta w_{n}=l+\sum_{i=n}^{\infty} v_{i} G\left(y_{\sigma(i)}\right)$

This implies

$\sum_{i=n}^{\infty} v_{i} G\left(y_{\sigma(i)}\right)<\infty, \quad$ for $n \geq n_{2}$.

From this, it follows, due to (E7), that $\liminf _{n \rightarrow \infty}\left(G\left(y_{\sigma(n)}\right) / n\right)=0$. Hence $\liminf _{n \rightarrow \infty}\left(y_{\sigma(n)} /\right.$ $n)=0$, by (E1) and (E9). As $\lim _{n \rightarrow \infty} \sigma(n)=\infty$ and $\sigma(n)>\gamma n$ for large $n$, due to (E11), we obtain 
International Journal of Mathematical, Engineering and Management Sciences

Vol. 5, No. 4, 663-681, 2020

https://doi.org/10.33889/IJMEMS.2020.5.4.054

$\liminf _{n \rightarrow \infty}\left(y_{n} / n\right)=0$

As $w_{n}>0$ and $\Delta w_{n}>0$, we can find $M_{0}>0$ such that $w_{n}>M_{0}$ for $n \geq n_{3} \geq n_{2}$. For any $0<\epsilon$, from (15) it follows due to (E3) and (E4) that $z_{n} \geq w_{n}-\epsilon$ for large $n$. It implies, by Remark 2.8 that $\exists M_{1}$, with $0<M_{1}<M_{0}$, and $y_{n}-p_{n}^{\{1\}} y_{n-m_{1}}-p_{n}^{\{2\}} y_{n-m_{2}}>M_{1}$ for $n \geq$ $n_{4}>n_{3}$. Using (R4) with $i=1$ or (R9) we have $p_{n}^{\{1\}}>1$. Hence one may obtain

$y_{n}>y_{n-r}+M_{1}, n \geq n_{4}$,

where $r=m_{1}$.

Let, $N_{0}>n_{4}, \quad M=\min \left\{y_{n}: N_{0} \leq n \leq N_{0}+r\right\}$ and $0<\beta<\min \left\{\frac{M}{\left(N_{0}+r\right)}, \frac{M_{1}}{2 r}\right\}$, define, for $n \geq N_{0}, A(n)=M_{1}-\beta r$.

Thus $A(n)>0$ for $n \geq N_{0}$. Since $y_{n} \geq M$ for $N_{0} \leq n \leq N_{0}+r$ and $\beta\left(N_{0}+r\right)<M$, then $y_{n}>\beta n$ for $N_{0} \leq n \leq N_{0}+r$ and $N_{0}+r \leq n \leq N_{0}+2 r$ implies $N_{0} \leq n-r \leq N_{0}+r$. Using (21), we obtain, for $N_{0}+r \leq n \leq N_{0}+2 r, y_{n}>y_{n-r}+M_{1} \geq \beta(n-r)+M_{1}>\beta n$,

then $\beta n<A(n)+\beta n=M_{1}+\beta(n-r)$. Using a simple induction we prove $y_{n}>\beta n$ for $n \geq$ $N_{0}$. Hence $\liminf _{n \rightarrow \infty}\left[y_{n} / n\right] \geq \beta>0$, a contradiction to (20). Thus, $\lambda$ is not equal to $\infty$. Further, if $\lambda$ is not equal to $-\infty$ then $\lambda \in R$. Then easily, we conclude that $\Delta w_{n}>0$ and $\lim _{n \rightarrow \infty} \Delta w_{n}=0$. The proof for the case $y_{n}<0$, eventually is similar. Therefore the lemma is proved.

\section{Sufficient Conditions}

In this section, it is investigated to find, sufficent conditions for all the non oscillatory solutions of (2), tending to zero.

Theorem 3.1 Let any one of the conditions (R1) or (R2) hold for $k=2$. Consider $p_{n}^{\{j\}}$ to satisfy $\liminf _{n \rightarrow \infty}\left|p_{n}^{j}\right|>0$ for (R2). If (E1), (E3), (E9) and (E11) hold, then any solution of (2) which does not oscillate, tends to zero as $n \rightarrow \infty$.

Proof: Suppose $y=y_{n}$ be a solution of (2) for $n \geq N_{1}$ which is non-oscillatory. Then $y_{n}>0$ or $y_{n}<0$. Suppose $y_{n}>0$ eventually. $\exists \mathrm{a}+\mathrm{ve}$ integer $n=n_{0}$ such that $y_{n}>0, y_{n-m_{1}}>$ $0, y_{n-m_{2}}>0$ and $y_{\sigma(n)}>0$ for $n \geq n_{0} \geq N_{1}$. For $n \geq n_{0}$, we set $z_{n}$ and $w_{n}$ as in (14) and (15) respectively, to obtain (16). Hence $w_{n}, \Delta w_{n}$ are monotonic and of one sign for $n \geq n_{1} \geq n_{0}$. Then $\lim _{n \rightarrow \infty} w_{n}=\lambda,-\infty \leq \lambda \leq+\infty$. We claim $y_{n}$ is bounded. Otherwise, $y_{n}$ is unbounded. Hence $\exists$ a sub-sequence $\left\{y_{n_{k}}\right\}$ such that

$n_{k} \rightarrow \infty, \quad y_{n_{k}} \rightarrow \infty$ as $\quad k \rightarrow \infty, \quad$ and $y\left(n_{k}\right)=\max \left\{y_{n}: n_{1} \leq n \leq n_{k}\right\}$

We may choose $n_{k}$ large enough so that for $n_{k}-r \geq n_{1}, \sigma\left(n_{k}\right) \geq n_{1}$, where $r=\max \left\{m_{1}, m_{2}\right\}$. Then by (E3), for $\epsilon>0$, we can find a +ve integer $n_{2}$ such that, for $k \geq n_{2} \geq n_{1}$ implies $\left|F_{n_{k}}\right|<\gamma$, for some constant $\gamma>0$. Hence for $k \geq n_{2}$, if (R1) holds, then we have $w_{n_{k}} \geq$ 
International Journal of Mathematical, Engineering and Management Sciences

Vol. 5, No. 4, 663-681, 2020

https://doi.org/10.33889/IJMEMS.2020.5.4.054

$y_{n_{k}}(1-b)-\gamma$

Similarly, if (R2) holds, then for $k \geq n_{2}$, we have $w_{n_{k}} \geq y_{n_{k}}-\gamma$.

Taking $k \rightarrow \infty$, for either case (R1) or (R2), we find $\lim _{n \rightarrow \infty} w_{n}=\infty$, because of the monotonic nature of $w_{n}$. Hence $w_{n}>0, \Delta w_{n}>0$ for $n \geq n_{2} \geq n_{1}$ and $\Delta^{2} w_{n} z 0$ and is in - ve. As $m=$ $2, \exists \mathrm{a}+$ ve integer $p=1$. by Lemma 2.4. Then $\lim _{n \rightarrow \infty} \Delta w_{n}=l$ (finite) exists. Application of Lemma 2.7 to (16), results (18). Consequently (19) follows. Because of (E2), the inequality (19) yields $\liminf _{n \rightarrow \infty} G\left(y_{\sigma(n)}\right)=0$ for $n \geq n_{3}$. Then we claim $\liminf _{n \rightarrow \infty} y_{\sigma(n)}=0$. Otherwise, there exists $n_{4} \geq n_{3}$ and $\gamma>0$ such that $n \geq n_{4}$ implies $y_{\sigma(n)}>\gamma$. By (E1) and (E9), we obtain $G\left(y_{\sigma(n)}\right)>\gamma \delta>0$, for $n \geq n_{4}$, contradiction Therefore, $\liminf _{n \rightarrow \infty} y_{\sigma(n)}=0$ As $\lim _{n \rightarrow \infty} \sigma(n)=\infty$, it follows that $\liminf _{n \rightarrow \infty} y_{n}=0$. Since $w_{n}>0$ and $\Delta w_{n}>0$, we choose $B>0$, such that $w_{n}>B$ for $n \geq n_{4} \geq n_{3}$. Then we claim,

$\liminf _{n \rightarrow \infty} \frac{y_{n}}{w_{n}}=0$

Otherwise, there exists $a>0$ such that eventually $y_{n}>a w_{n}>a B$ which implies $\liminf _{n \rightarrow \infty} y_{n} \geq a B>0$, a contradiction to $\liminf _{n \rightarrow \infty} y_{\sigma(n)}=0$. Set, for $n \geq n_{4}$,

$a_{n}^{\{1\}}=p_{n}^{\{1\}} \frac{w_{n-m_{1}}}{w_{n}}$ and $a_{n}^{\{2\}}=p_{n}^{\{2\}} \frac{w_{n-m_{2}}}{w_{n}}$

It is clear from (E3) and $\lim _{n \rightarrow \infty} w_{n}=\infty$, that $\lim _{n \rightarrow \infty} \frac{F_{n}}{w_{n}}=0$.

Then we have

$$
\begin{aligned}
1 & =\lim _{n \rightarrow \infty}\left[\frac{w_{n}}{w_{n}}\right] \\
& =\lim _{n \rightarrow \infty}\left[\frac{y_{n}-p_{n}^{\{1\}} y_{n-m_{1}}-p_{n}^{\{2\}} y_{n-m_{2}}-F_{n}}{w_{n}}\right] \\
& =\lim _{n \rightarrow \infty}\left[\frac{y_{n}}{w_{n}}-\frac{a_{n}^{\{1\}} y_{n-m_{1}}}{w_{n-m_{1}}}-\frac{a_{n}^{\{2\}} y_{n-m_{2}}}{w_{n-m_{2}}}-\frac{F_{n}}{w_{n}}\right] \\
& =\lim _{n \rightarrow \infty}\left[\frac{y_{n}}{w_{n}}-\frac{a_{n}^{\{1\}} y_{n-m_{1}}}{w_{n-m_{1}}}-\frac{a_{n}^{\{2\}} y_{n-m_{2}}}{w_{n-m_{2}}}\right] .
\end{aligned}
$$

Since $\left\{w_{n}\right\}$ is a increasing sequence, then $\frac{w_{n-m_{j}}}{w_{n}}<1$ for $j=1,2$. For $j=1,2$ if $p_{n}^{\{j\}}$ is defined as in (R1) then $0 \leq a_{n}^{\{j\}}<p_{n}^{\{j\}} \leq b<1$. However, if $p_{n}^{\{j\}}$ is defined as in (R2) then $0 \geq a_{n}^{\{j\}} \geq$ $p_{n}^{\{j\}} \geq-b_{j}>-1$. Hence it is clear that for $j=1,2$, if $p_{n}^{\{j\}}$ satisfies (R1) or (R2) then $a_{n}^{\{j\}}$ also satisfies the corresponding conditions (R1) or (R2) accordingly. If (R1) holds then Lemma 2.12(a) yields, due to (23), that $\lim _{n \rightarrow \infty}\left[\frac{y_{n}}{w_{n}}-\frac{a_{n}^{\{1\}} y_{n-m_{1}}}{w_{n-m_{1}}}-\frac{a_{n}^{\{2\}} y_{n-m_{2}}}{w_{n-m_{2}}}\right] \leq 0$, a contradiction to (24). Again if (R2) holds then by Lemma $2.15 \lim _{n \rightarrow \infty}\left[\frac{y_{n}}{w_{n}}-\frac{a_{n}^{\{1\}} y_{n-m_{1}}}{w_{n-m_{1}}}-\frac{a_{n}^{\{2\}} y_{n-m_{2}}}{w_{n-m_{2}}}\right]=0$, a contradiction to (24). 
Hence $\left\{y_{n}\right\}$ is bounded. Then $z_{n}$ and $w_{n}$ are bounded. By (E3), (E4) and monotonic nature of $w_{n}$ we obtain $\lim _{n \rightarrow \infty} w_{n}=\lim _{n \rightarrow \infty} z_{n}=\lambda$ (finite). We claim $\liminf _{n \rightarrow \infty} y_{n}=0$. Apply Lemma 2.7 to (16), to get

$w_{n}=\lambda-\sum_{i=n}^{\infty}(i-n+1) v_{i} G\left(y_{\sigma(i)}\right)$,

for $n \geq n_{1}$, where $n_{1}$ is some large + ve integer. Therefore,

$\sum_{i=n}^{\infty}(i-n+1) v_{i} G\left(y_{\sigma(i)}\right)<\infty, \quad n \geq n_{1}$.

Use Lemma 2.9 and Remark 2.10 in the inequality (26), to get

$\sum_{i=n}^{\infty} i v_{i} G\left(y_{\sigma(i)}\right)<\infty, \quad n \geq n_{1}$.

The inequality (27), due to (E7) yields $\liminf _{n \rightarrow \infty} G\left(y_{\sigma(n)}\right)=0$. Since $\lim _{n \rightarrow \infty} \sigma(n)=\infty$, it can be easily shown that $\liminf _{n \rightarrow \infty} G\left(y_{n}\right)=0$. This implies due to (E1) and (E9) that $\liminf _{n \rightarrow \infty} y_{n}=$ 0 . From Lemma 2.12, it follows that $\lim _{n \rightarrow \infty} z_{n}=0$ and $\lim _{n \rightarrow \infty} y_{n}=0$.

Next, if $y_{n}<0$, is a solution of (2) for large $n$, then we put $x_{n}=-y_{n}$ to obtain $x_{n}>0$ and then (2) reduces to

$\Delta^{2}\left(x_{n}-p_{n}^{\{1\}} x_{n-m_{1}}-p_{n}^{\{2\}} x_{n-m_{2}}\right)+v_{n} \tilde{G}\left(x_{\sigma(n)}\right)=\tilde{f}_{n}$,

where

$\tilde{f}_{n}=-f_{n}, \tilde{G}(v)=-G(-v)$.

Further,

$\tilde{F}_{n}=-F_{n} \quad$ implies $\quad \Delta^{2}\left(\tilde{F}_{n}\right)=\tilde{f}_{n}$.

Taking the above facts into consideration, the following conditions can be verified to hold.

- $x \tilde{G}(x)>0$ for $x \neq 0$.

- For $u>0$ there exists $\delta>0$ such that $\tilde{G}(u) \geq \delta u$. For $u<0$ there exists $\delta>0$ such that $\tilde{G}(u) \leq \delta u$.

- $\quad \exists$ a sequence $\left\{\tilde{F}_{n}\right\}$ which is bounded, $\Delta^{2}\left(\tilde{F}_{n}\right)=\tilde{f}_{n}$ and $\lim _{n \rightarrow \infty} \tilde{F}_{n}=0$.

Rest of the proof follows on similar lines as above, hence the proof is complete.

From the above theorem the following corollary follows.

Corollary 3.2 Solution of (2) which are unbounded, oscillate under the assumptions of Theorem 3.1.

Remark 3.3 Corollary 3.2 extends (Rath and Behera, 2018) [Theorem 1, Theorem 2] to second order . 
International Journal of Mathematical, Engineering and Management Sciences

Vol. 5, No. 4, 663-681, 2020

https://doi.org/10.33889/IJMEMS.2020.5.4.054

Theorem 3.4 Consider $p_{n}^{\{j\}}$ to satisfy one of the conditions (R1)-(R4) for $k=2$. If (E1), (E3),(E4) and (E7) hold good, then non oscillatory bounded solutions of (2) tend to zero as $n \rightarrow \infty$.

Proof: Suppose $y=y_{n}$ be a solution of (2) which is bounded for $n \geq N_{1}$. If it fails to oscillate then eventually $y_{n}>0$ or $y_{n}<0 . \exists$ a +ve integer $n_{0}$ such that $y_{n}>0, y_{n-m_{1}}>0, y_{n-m_{2}}>$ $0, y_{\sigma(n)}>0$ for $n \geq n_{0} \geq N_{1}$. Set $z_{n}$ and $w_{n}$ as in (14), and (15) respectively, to obtain (16). Then $w_{n}, \Delta w_{n}$ are monotonic and of one sign for $n \geq n_{1} \geq n_{0}$. Since $y_{n}$ is bounded, $z_{n}$ and $w_{n}$ are bounded. Using (E3), (E4) and monotonic bahaviour of $w_{n}$, we get $\lim _{n \rightarrow \infty} z_{n}=\lim _{n \rightarrow \infty} w_{n}=$ $\lambda$. It exists finitely. Now apply Lemma 2.7 to (16), to get (25) and (26) for $n \geq n_{2}>\mathrm{n}_{1}$, where $n_{2}>0$ is some large integer. By using Lemma 2.9 and Remark 2.10 in the equation (26), we get (27). The inequality (27), due to (E7) yields $\liminf _{n \rightarrow \infty} G\left(y_{\sigma(n)}\right)=0$. Since $\lim _{n \rightarrow \infty} \sigma(n)=\infty$, it can be easily shown that $\liminf _{n \rightarrow \infty} G\left(y_{n}\right)=0$. This implies due to (E1) that $\liminf _{n \rightarrow \infty} y_{n}=0$. From Lemma 2.12, it follows that $\lim _{n \rightarrow \infty} z_{n}=0$ and $\lim _{n \rightarrow \infty} y_{n}=0$. If $y_{n}$ is eventually -ve , then as in the proof of the theorem 3.1, we may move with $x_{n}=-y_{n}\left(x_{n}\right.$ is a positive solution of (28)) to prove $\lim _{n \rightarrow \infty} \mathrm{x}_{n}=0$, hence, the theorem is complete.

Remark 3.5 All type of $G$, be it linear, sublinear or super linear, are accomodated in theorem 3.4 . It improves, extends, generalize the sufficient part of the theorem due to (Parhi and Tripathy, 2003) [Theorem 2.8].

Theorem 3.6 Suppose that (R6) holds. Assume that $\sigma\left(n-m_{j}\right)=\sigma(n)-m_{j}$ for $j=1,2$. Let (E1), (E3)-(E6), (E9)-(E11) hold. Then non oscillatory solutions of (2) tend to zero as $n \rightarrow \infty$.

Proof: Consider an eventually +ve solution $y=\left\{y_{n}\right\}$ of (2) for $n \geq n_{0} \geq N_{1}$. Then set $z_{n}$, and $w_{n}$ as in (14) and (15) respectively to get (16) for $n>n_{1} \geq n_{0}$. Hence $w_{n}, \Delta w_{n}$ are monotonic and of one sign for $n \geq n_{1}$. Then (17) holds by (E3), (E4), which implies

$\lim _{n \rightarrow \infty} w_{n}=\lim _{n \rightarrow \infty} z_{n}=\lambda, \quad \lambda \in[-\infty, \infty]$

If $\lambda$ is $-v e$, then $z_{n}<0$, for very large $n$, which is a contradiction. If $\lambda$ is equal to 0 , then $y_{n} \leq z_{n}$, implies $\lim _{n \rightarrow \infty} y_{n}=0$. If $\lambda>0$, then $w_{n}>0$ for $n \geq n_{2}$. As $\mathrm{m}=2$, by Lemma 2.4 , we have $p=1$, and this implies $w_{n}>0, \Delta w_{n}>0$. Hence $\lim _{n \rightarrow \infty} \Delta w_{n}=l$ exists. Note that, $\lambda \in(0, \infty) \Rightarrow p=0$, a contrdiction. Hence $\lambda=\infty$. Application of Lemma 2.7 to (16), yields (18) and consequently (19) holds. Using Lemma 2.9 and Remark 2.10, we get,

$\sum_{i=N_{2}}^{\infty} v_{i} G\left(y_{\sigma(i)}\right)<\infty, N_{2} \geq n_{2}$

Putting $i=j-m_{1}$, one may get

$\sum_{j=N_{2}+m_{1}}^{\infty} v_{j-m_{1}} G\left(y_{\sigma\left(j-m_{1}\right)}\right)<\infty$.

As $0 \leq-p_{n}^{\{j\}} \leq b_{j}, j=1,2$ then by (E0) and (E1), one has $G\left(-p_{\sigma(j)}\right) \leq c$. Since $v_{j}^{*} \leq v_{j-m_{i}}$ for $i=1,2$ then using Lemma 2.9 and $\sigma\left(j-m_{i}\right)=\sigma(j)-m_{i}$ for $i=1,2$, it follows from the above inequality that 
$\sum_{j=N_{3}}^{\infty} v_{j}^{*} G\left(-p_{\sigma(j)}^{\{1\}}\right) G\left(y_{\sigma(j)-m_{1}}\right)<\infty$.

Then using (E13), one obtains

$\sum_{j=N_{3}}^{\infty} v_{j}^{*} G\left(-p_{\sigma(j)}^{\{1\}} y_{\sigma(j)-m_{1}}\right)<\infty$

Following the line of argument that (32) is obtained from (31), one also finds

$\sum_{j=N_{3}}^{\infty} v_{j}^{*} G\left(-p_{\sigma(j)}^{\{2\}} y_{\sigma(j)-m_{2}}\right)<\infty$

From (31) and the fact that $v_{n} \geq v_{n}^{*}$, it follows that

$\sum_{j=N_{3}}^{\infty} v_{j}^{*} G\left(y_{\sigma(j)}\right)<\infty$

Further, use of (E13), (32), (33) and (34), yields

$\beta \sum_{i=N_{3}}^{\infty} v_{i}^{*} G\left(z_{\sigma(i)}\right)<\infty$

Since $m=2$ then by Lemma 2.4 we have $p=1$, hence there exists $A>0$ such that $w_{n}>A$ for $n \geq N_{4} \geq N_{3}$. For any $\epsilon>0$, using (E3), (E4), we obtain $z_{n} \geq w_{n}-\epsilon$, for $n \geq N_{5} \geq N_{4}$. Thus, due to Remark 2.8, we can find $0<B<A$ such that

$z_{n}>B \quad$ for $n \geq N_{6} \geq N_{5}$.

By (E11), we have $\sigma(n) / n>b>0$ for $n \geq N_{7} \geq N_{6}$. Subsequent use of (36), (E5) and (E9) implies

$\sum_{i=N_{7}}^{\infty} v_{i}^{*} G\left(z_{\sigma(i)}\right) \geq B \delta \sum_{i=N_{8}}^{\infty} v_{i}^{*}=\infty$,

which is a contradiction because of (35). Therefore, the proof is complete for the case $y_{n}>0$.

If $y_{n}<0$, for some large $n$, then one may go ahead as in the proof of theorem 3.1, by the substitution $x_{n}=-y_{n}$ and note that, $x_{n}>0$, is a solution of (28) with (29) and (30). One may further observe that , $G=\tilde{G}$ by (E10). Taking note of the above facts and following the proof for the case when $y_{n}$ is $+v e$, as above, one may prove that $\lim _{n \rightarrow \infty} x_{n}=0$, which yields $\lim _{n \rightarrow \infty} y_{n}=0$ and this proves the theorem.

Note that the above result even holds, for (R10) instead of (R6).

Remark 3.7 Theorem 3.6 extends, improves and generalizes the sufficiency part of the Theorem 2.6 of (Parhi and Tripathy, 2003). 
International Journal of Mathematical, Engineering and Management Sciences

Vol. 5, No. 4, 663-681, 2020

https://doi.org/10.33889/IJMEMS.2020.5.4.054

Remark 3.8 The function $G(u)=\left(\beta+|u|^{\mu}\right)|u|^{\delta}\{\operatorname{sgn}\}(u)$, for $\delta>0, \mu>0, \delta+\mu \geq 1, \beta \geq$ 1 satisfies (E1) (E6), (E9) and (E10) which could be proved by using the well known inequality (Hilderbrandt, 1963, p.292)

$u^{p}+v^{p} \geq \begin{cases}(u+v)^{p}, & 0 \leq p<1 \\ 2^{1-p}(u+v)^{p}, & p \geq 1\end{cases}$

Remark 3.9 The condition $\sum_{n=N_{1}}^{\infty} v_{n}^{*}=\infty$, implies (E2).

Theorem 3.10 Suppose that (R6) holds. Assume that $\sigma\left(n-m_{j}\right)=\sigma(n)-m_{j}$ for $j=1,2$. Let (E1)- (E4), (E6), (E9)- (E11) hold and $v_{n}$ is monotonic. Then any solution of (2) tends to zero as $n \rightarrow \infty$ or oscillates .

Proof: This proof follows from the proof of theorem 3.6 by the following consideration. We claim if $v_{n}$ is monotonic then both (E2) and (E5) are equivalent. Obviously, if $v_{n}$ is non increasing then $v_{n}^{*}=v_{n}$. As a result, the equivalence of (E2) and (E5) is evident. Further, if $v_{n}$ is non decreasing, then assume that (E2) holds good. Then $v_{n}^{*}=v_{n-r}$, where $r=\max \left\{m_{1}, m_{2}\right\}$. Hence $\sum_{n=N_{1}}^{\infty} v_{n}^{*}=\sum_{n=N_{1}}^{\infty} v_{n-r}=\sum_{j=N_{1}-r}^{\infty} v_{j}=\infty$ by Lemma 2.9. Hence (E5) holds. Thus, (E2) and (E5) are equivalent, when $v_{n}$ is monotonic and the proof is complete.

Theorem 3.11 Consider the second order NDDE

$\Delta^{2}\left(y_{n}-\sum_{j=1}^{k} p_{n}^{\{j\}} y_{n-m_{j}}\right)+v_{n} G\left(y_{\sigma(n)}\right)=0$

Suppose that $p_{n}^{\{j\}}$ satisfies the condition (R4) with $k=2$. Let (E1),(E3), (E4), (E7),(E9) and (E11) hold. Then

(i) all non oscillatory solutions $y_{n}$ of (38), which are bounded, tend to zero, as $n \rightarrow \infty$,

(ii) all non oscillatory solutions $y_{n}$ of (38), which are unbounded satisfies

$\lim _{n \rightarrow \infty}\left|\left(y_{n-m_{1}}+y_{n-m_{2}}\right)\right|=\infty$. or $\liminf _{n \rightarrow \infty}\left|y_{n}\right|=0$.

Proof. Let $y_{n}$ be a eventually positive solution of (38) in some interval $\left[n_{1}, \infty\right)$. Then defining $z_{n}$ as in (14) we obtain

$\Delta^{2} z_{n}=-v_{n} G\left(y_{\sigma(n)}\right) \leq 0$.

From this, it follows that $z_{n}, \Delta z_{n}$ are monotonic and of constant sign on some interval $\left[n_{1}, \infty\right)$. Let us prove (A) and assume $y_{n}$ to be bounded. Then applying Lemma 2.17 with $f_{n} \equiv 0$, we have $\lim _{n \rightarrow \infty} z_{n}=\lambda$. Since $y_{n}$ is bounded, $\lambda=-\infty$ is not possible. Hence $\lambda$ is finite. Then apply Lemma 2.7 to (39), to get

$z_{n}=\lambda-\sum_{i=n}^{\infty}(i-n+1) v_{i} G\left(y_{\sigma(i)}\right)$

Consequently (26) and (27) hold. The inequality (27), because of (E7) implies $\liminf _{n \rightarrow \infty} G\left(y_{\sigma(n)}\right)=0 . \lim _{n \rightarrow \infty} \sigma(n)=\infty$, it can be easily shown that $\liminf _{n \rightarrow \infty} G\left(y_{n}\right)=0$. This implies due to (E1) and continuity of G that $\liminf _{n \rightarrow \infty} y_{n}=0$. Then applying Lemma 2.12, 
International Journal of Mathematical, Engineering and Management Sciences

Vol. 5, No. 4, 663-681, 2020

https://doi.org/10.33889/IJMEMS.2020.5.4.054

we obtain $\lim _{n \rightarrow \infty} y_{n}=0$. Next let us proceed to prove (B) and consider $y_{n}$ to be positive solution of (38) which is unbounded in some interval $\left(n_{1} \infty\right)$. Then by Lemma 2.17 it follows either $\lim _{n \rightarrow \infty} z_{n}=\lambda$ (finite) or $\lim _{n \rightarrow \infty} Z_{n}=-\infty$. If the latter holds then Since $p_{n}^{\{j\}}$ for $j=1,2$ are bounded, there exists a positive scalar $b$ such that $0<p_{n}^{\{j\}}<b$. From (14) it follows that

$z_{n}=y_{n}-p_{n}^{\{1\}} y_{n-m_{1}}-p_{n}^{\{2\}} y_{n-m_{2}} \geq-b y_{n-m_{1}}-b y_{n-m_{2}}$,

This implies $y_{n-m_{1}}+y_{n-m_{2}} \geq \frac{z_{n}}{-b} \rightarrow+\infty$ as $n \rightarrow \infty$. So, $\lim _{n \rightarrow \infty}\left(y_{n-m_{1}}+y_{n-m_{2}}\right)=+\infty$.

If the former holds then proceeding as in part (a) of the proof one may obtain $\liminf _{n \rightarrow \infty} y_{n}=0$. For the case when $y_{n}<0$ for $n \geq n_{0}$, the proof is similar. Thus, the theorem is proved.

Theorem 3.12 Let (R4) with $k=2$, hold. Suppose (E1), (E3),(E4), (E8), (E9) and (E11) hold good.

Then

(i) non oscillatory bounded solutions $y_{n}$ of (2), tend to zero as $n \rightarrow \infty$,

(ii) non oscillatory unbounded solutions $y_{n}$ of (2), satisfy $\lim _{n \rightarrow \infty}\left(y_{n-m_{1}}+y_{n-m_{2}}\right)=+\infty$.

Proof: Clearly, (E8) implies (E7). Then proof of (i) follows from, proof of Theorem 3.4 for case (R4). Now to prove (ii), assume $y=\left\{y_{n}\right\}$ be a +ve solution of (2) which is unbounded. By virtue of Lemma 2.17, one is to get $\lim _{n \rightarrow \infty} w_{n}=\lambda$ (finite) or $\lim _{n \rightarrow \infty} w_{n}=-\infty$. In this situation we claim $\lim _{n \rightarrow \infty} w_{n}=\lambda$, cannot hold. Else, apply Lemma 2.7 to (16) to obtain (25) and (26) and then use Lemma 2.9 and remark 2.10 to show that (27) holds. As $y_{\sigma(n)}$ is unbounded, we find a sub-sequence $\left\{\sigma\left(n_{j}\right)\right\}$ of $\{\sigma(n)\}$ such that $y_{\sigma\left(n_{j}\right)}>\zeta>0$, for $j>n_{1}$. Hence using (E8) and (E9), we have

$\sum_{j=n_{1}}^{\infty}\left(n_{j}\right) v_{n_{j}} G\left(y_{\sigma\left(n_{j}\right)}\right)>\zeta \delta \sum_{j=n_{1}}^{\infty}\left(n_{j}\right) v_{n_{j}}=\infty$

a contradiction to (27). Thus $\lim _{n \rightarrow \infty} w_{n}=-\infty$. We observe that (17) holds because of (E3), (E4). Hence $\lim _{n \rightarrow \infty} z_{n}=-\infty$. Since $p_{n}^{\{j\}}$ for $j=1,2$ are bounded then there exists a positive scalar $b$ such that $0<p_{n}^{\{j\}}<b$. From (14) it follows that

$z_{n}=y_{n}-p_{n}^{\{1\}} y_{n-m_{1}}-p_{n}^{\{2\}} y_{n-m_{2}} \geq-b y_{n-m_{1}}-b y_{n-m_{2}}$.

This implies $y_{n-m_{1}}+y_{n-m_{2}} \geq \frac{z_{n}}{-b} \rightarrow+\infty$ as $n \rightarrow \infty$. So, $\lim _{n \rightarrow \infty}\left(y_{n-m_{1}}+y_{n-m_{2}}\right)=+\infty$.

For the case, when $y_{n}$ is - ve for large $n$, the proof is similar and this is the end of the proof.

Before, this article gets closed, some examples are given to illustrate the outcomes. 
International Journal of Mathematical, Engineering and Management Sciences

Vol. 5, No. 4, 663-681, 2020

https://doi.org/10.33889/IJMEMS.2020.5.4.054

Example 3.13 Consider the NDDE

$\Delta^{2}\left(y_{n}+\frac{1}{4} y_{n-1}+\frac{1}{8} y_{n-2}\right)+n^{-2} y_{n-3}^{\alpha}=\frac{1}{2^{n+1}}+\frac{2^{3 \alpha}}{2^{\alpha n} n^{2}}$

where $n \geq 3 G(x)=x^{\alpha}, \alpha$ is + ve and is the quotient of two odd integers. Here, $p_{n}^{\{j\}}$ satisfies (R2) and $v_{n}=n^{-2}, f_{n}=\frac{1}{2^{n+1}}+\frac{2^{3 \alpha}}{2^{\alpha n} n^{2}}$. Easily, we can verify that, $\sum_{n=n_{0}}^{\infty} \mathrm{n} f_{n}<\infty$ and all the conditions of Theorem 3.1 are satisfied by the equation (41). Hence $y_{n}=2^{-n}$ is a solution of (41), tending to zero as $n \rightarrow \infty$. Here $G$ could be linear, super linear, or sublinear,

Example 3.14 Consider the NDDE

$\Delta^{2}\left(y_{n}-\frac{1}{4} y_{n-1}-\frac{1}{8} y_{n-2}\right)+n^{-1} y_{n-3}^{\alpha}=\frac{2^{3 \alpha}}{2^{\alpha n_{n}}}$

where $n \geq 3, G(x)=x^{\alpha}, \alpha>1$ is the quotient of two integers, which are odd. Here, $p_{n}^{\{j\}}$ satisfies (R1) and $v_{n}=n^{-1}, f_{n}=\frac{2^{3 \alpha}}{2^{\alpha n} \mathrm{n}}$. Easily, we can verify that, $\sum_{n=n_{0}}^{\infty} \mathrm{n} f_{n}<\infty$ and all the conditions of Theorem 3.4 are satisfied by the equation (42). Therefore the solution $y_{n}=2^{-n}$ of (42), tends to zero as $n \rightarrow \infty$.

\section{Conclusion}

This paper, investigates to establish that the condition (E2) or (E7) is sufficient, for every solution of (2) to be oscillatory or tending to zero. Theorem 3.11 is obtained under (E7), which is less restrictive than (E2). The condition " $G$ is non decreasing," which is very often used for non linear neutral equations, is relaxed in this work. As a result, the theorems 3.11 and 3.12 extend and generalize the sufficiency part of the theorems 2.8 and 2.7 of (Parhi and Tripathy, 2003) respectively. Further, the results extend (Rath and Behera, 2018) to $2^{\text {nd }}$ order NDDE. At the end, the following open problems are proposed to the reader, which might be helpful for further research.

Problem 4.1 It would be interesting to prove theorem 3.12, with (R5) instead of (R4) and under the hypothesis, (E8) or, a condition weaker than (E8).

Problem 4.2 If $v_{n}$ changes sign, under the consideration of $G(x)=x$ or $G(x) \neq x$, then one should investigate to find the sufficient conditions for the qualitative behaviour of (1) or that of an equation of order $m>2$.

\section{Conflict of Interest}

The authors declare that this publication is not subject to conflict of interest.

\section{Acknowledgment}

This research did not receive any funding from any agencies. The authors extend their gratitude to the reviewers and editors, for their helpful comments and suggestions to improve the presentation of the paper. 
International Journal of Mathematical, Engineering and Management Sciences

Vol. 5, No. 4, 663-681, 2020

https://doi.org/10.33889/IJMEMS.2020.5.4.054

\section{References}

Agarwal, R.P., \& Grace, S.R. (1999). Oscillation of higher order nonlinear difference equations of neutral type. Applied Mathematics Letters, 12 , 77-83.

Agarwal, R.P., Manuel, M.M.S., \& Thandapani, E. (1996). Oscillatory and nonoscillatory behavior of second order neutral delay difference equations. Mathematical and Computer Modelling, 24(1), 5-11.

Gyori, I., \& Ladas, G. (1991). Oscillation theory of delay differential equations with applications. Clarendon Press, Oxford.

Hilderbrandt, T.H. (1963). Introduction to the theory of integration. Academic Press, New-York.

Karpuz, B., Ocalan, O., \& Yildiz, M.K. (2009a). Oscillation of a class of difference equations of second order. Mathematical and Computer Modelling, 49(5-6), 912-917.

Karpuz, B., Rath, R.N., \& Rath, S.K. (2009b). On Oscillation and asymptotic behavior of a higher order functional difference equation of neutral type. International Journal of Difference Equations 4(1), 69-96.

Kelle, W.G., \& Peterson, A.C. (1991). Difference equations: An introduction with applications. Academic Press, New York.

Malik, S.C., \& Arora, S. (2008). Mathematical analysis. New Age International (P) Ltd. Publishers (Third Edition), New-Delhi.

Parhi, N., \& Tripathy, A.K. (2003). Oscillation of forced nonlinear neutral delay difference equations of first order. Czechoslovak Mathematical Journal, 53(1), 83-101

Rath, R., \& Behera, C. (2018). Oscillatory and asymptotic behaviour of a first order neutral equation of discrete type with variable several delay under $\Delta$ sign. International Journal of Mathematics and Mathematical Sciences, 2018, Article ID 4586176, https//doi.org10.1155/20184586176.

Rath, R.N., \& Padhy, L.N. (2005). Necessary and sufficient conditions for oscillation of solutions of a first order forced nonlinear difference equation with several delays. Fasciculi Mathematici, 35 , 99-113.

Rath, R.N., Barik, B.L.S. \& Rath, S.K. (2010). Oscillation of higher order neutral Functional Difference Equations with positive and negative coefficients. Mathematica Slovaca, 60(3), 361-384.

Royden, H.L. (1988). Real analysis. Macmillan Publishing Company (3rd Edition), New York.

Thandapani, E., Manuel, M., Graef, J.R., \& Spikes, P.W. (1999). Oscillation of a higher order neutral difference equation with a forcing term. International Journal of Mathematics and Mathematical Sciences, 22(1), 147-154.

Yildiz, M.K., \& Ocalan, O. (2007). Oscillation results for higher order non-linear neutral delay difference equations. Applied Mathematics Letter, 20, 243-247.

Yildiz, M.K. (2015). Oscillation of a class of nonlinear difference equations of second order with oscillating coefficients. Konuralp Journal of Mathematics, 3(2), 211-218.

Yildiz, M.K., Karpuz , B., \& Ocalan, O. (2009). Oscillation of nonlinear neutral delay differential equations of second-order with positive and negative coefficients. Turkish Journal of Mathematics, 33(4), $341-350$

Zhou, Y., \& Huang, Y.Q. (2003). Existence for nonoscillatory solutions of higher-order nonlinear neutral difference equations. Journal of Mathematical Analysis and Applications, 280(1), 63-76.

Original content of this work is copyright (C) International Journal of Mathematical, Engineering and Management Sciences. Uses under the Creative Commons Attribution 4.0 International (CC BY 4.0) license at https://creativecommons.org/licenses/by/4.0/ 